\title{
Parallelized cytoindentation using convex micropatterned surfaces
}

\author{
Bojing Jia' ${ }^{1,2}$, Tse-Luen (Erika) Wee,3,4, Colton G. Boudreau³, Daniel J. Berard¹, Adiel Mallik,6,7, \\ David Juncker ${ }^{5,6,7}$, Claire M. Brown ${ }^{3,4, *}$, and Sabrina R. Leslie ${ }^{1, *}$ \\ ${ }^{1}$ Department of Physics, ${ }^{2}$ Department of Biology, ${ }^{3}$ Department of Physiology, ${ }^{4} L i f e$ Sciences Complex \\ Advanced Biolmaging Facility (ABIF), ${ }^{5}$ Department of Neurology and Neurosurgery, ${ }^{6}$ Department of \\ Biomedical Engineering, McGill University, Montreal, Quebec Canada, and ${ }^{7}$ Mc Gill University and Genome \\ Quebec Innovation Centre, Montreal, Quebec, Canada
}

${ }^{*}$ C.M.B. and S.R.L. contributed equally to this work.

BioTechniques 61:73-82 (August 2016) doi 10.2144/000114436

Keywords: cytoindentation, cell loading; confocal microscopy; convex lens-induced confinement; microfabrication

Here we present a high-throughput, parallelized cytoindentor for local compression of live cells. The cytoindentor uses convex lens-induced confinement (CLiC) to indent micrometer-sized areas in single cells and/or populations of cells with submicron precision. This is accomplished using micropatterned poly(dimethylsiloxane) (PDMS) films that are adhered to a convex lens to create arrays of extrusions referred to here as "posts." These posts caused local deformation of subcellular regions without any evidence of cell lysis upon CLiC indentation. Our micropost arrays were also functionalized with glycoproteins, such as fibronectin, to both pull and compress cells under customized confinement geometries. Measurements of Chinese hamster ovary (CHO-K1) cell migration trajectories and oxidative stress showed that the CLiC device did not damage or significantly stress the cells. Our novel tool opens a new area of investigation for visualizing mechanobiology and mechanochemistry within living cells, and the high-throughput nature of the technique will streamline investigations as current tools for mechanically probing material properties and molecular dynamics within cells, such as traditional cytoindentors and atomic force microscopy (AFM), are typically restricted to single-cell manipulation.

Recent interest in the mechanical properties of cells and forcedependent cellular dynamics has inspired the development of a number of mechanical tools to probe live cells. Cells subject to high mechanical stress, such as chondrocytes, osteocytes, and myocytes, are the focus of studies on tissue remodeling $(1,2,3)$. Inside the cell, structures such as the cytoskeleton and cellular anchors called focal adhesions reconfigure at a molecular level in response to mechanical cues $(4,5,6)$. Measuring the global viscoelastic properties of cells and gauging intracellular responses to mechanical perturbation require sensitive devices with the precision to locally perturb cells without lysis.

Existing strategies such as atomic force microscopy (AFM) and cytoindentors are restricted to single-cell manipulation. AFM probes the chemomechanical response of single cells with a beaded cantilever maneuvered by a set of piezo translation stages to indent cells one-by-one (7). Similarly, cytoindentors use piezo actuators to move a single glass probe to deform cells (8).

Biological samples are inherently highly variable, thus many experiments must be conducted on many cells in order to acquire sufficient statistical power to understand the behavior of cell populations and

\section{METHOD SUMMARY}

Here we present a high-throughput, precision cytoindentor that integrates convex lens-induced confinement (CLiC) microscopy within a live-cell incubator to enable simultaneous manipulation of multiple cells in combination with high-resolution microscopy. Micropatterned arrays of polydimethylsiloxane (PDMS) posts adhered to the convex lens locally deformed subcellular regions without cell lysis, enabling direct visualization of mechanochemistry and mechanotransduction. 
validate hypotheses. This often makes sequential cell-by-cell data collection time-consuming and laborious. The availability of an inexpensive, easyto-construct device that is able to indent cells in a parallel fashion would greatly improve experimental fidelity and streamline data acquisition.

Danilowicz et al. demonstrated a modified magnetic tweezer setup capable of simultaneously maneuvering multiple single-molecule complexes, but they have yet to use the technique on live cells (9). Single-cell micropipette aspiration, which applies a negative pressure to the cell and constricts the cell membrane through an orifice, has been applied in a parallel manner to characterize mechanophenotypes of single cells in suspension (10). However, micropipette aspiration can be challenging to apply to adherent cells. Sasoglu et al. proposed a microfabrication technique to produce arrays of poly(dimethylsiloxane) (PDMS) cylindrical posts, which can be maneuvered by micromanipulators to simultaneously indent multiple adhered cells (11). However, to our knowledge, no parallel cell perturbation device has been demonstrated on live cells.

Here, we adapt a single-molecule imaging technique, convex lensinduced confinement (CLiC), for high-throughput parallel cytoindentation. The live-cell CLiC platform was integrated within a stage-top microscope incubator for easy adaptation to a wide variety of microscope platforms, and PDMS micropost arrays enabled local loading of force (Figure 1). The device is an extension of previously published in vitro CLiC instruments that we developed to image biomolecules in aqueous solution $(12,13)$. It functions with a precision z-axis stepper motor to lower a plano-convex "CLiC-lens" into contact with the cell surface and gradually deform it mechanically (Figure 1A). To enable local deformation of cells on a customized, sub-cellular length scale $(\mu \mathrm{m})$, a PDMS film patterned with microposts was designed (Figure 1B) and adhered to the outer CLiC lens surface (Figure 1A). The cellular compression imposed by the microposts was greatest at the center of the lens and decreased radially away from the center (Figure 1A). PDMS
A

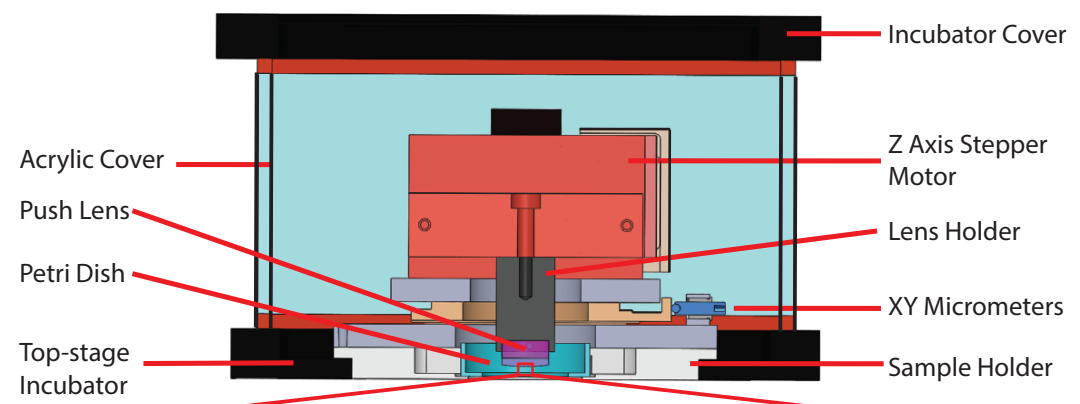
cubator

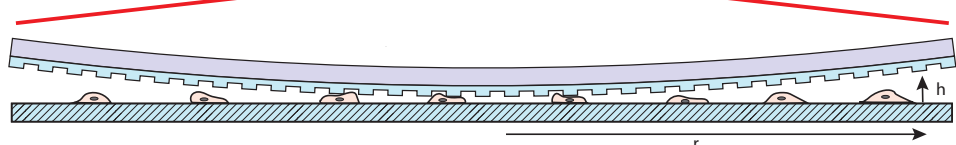

B

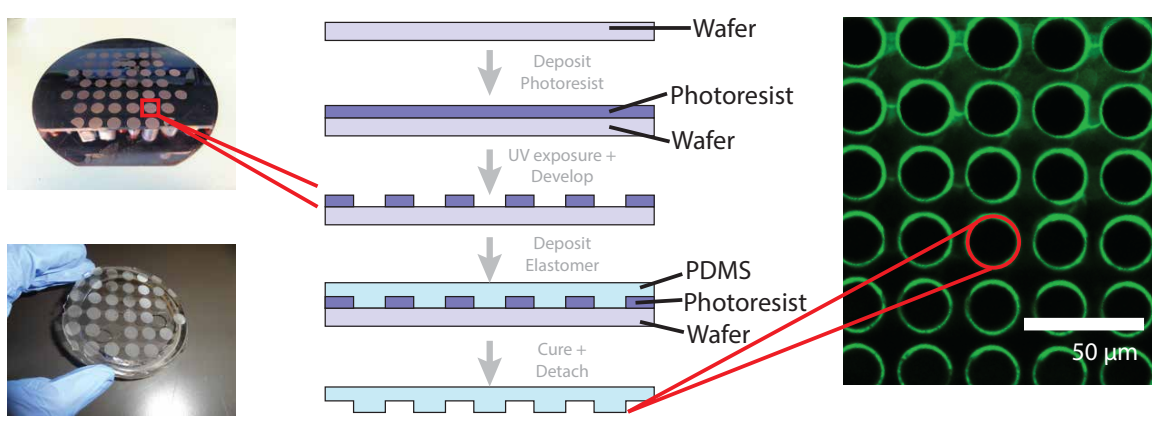

Figure 1. Illustration of the convex lens-induced confinement (CLiC) cytoindentor with polydimethylsiloxane (PDMS) posts. (A) A custom designed, remotely controlled CLiC cytoindentation device interfacing with a commercial live-cell incubation unit. (B) Schematic of PDMS post-fabrication. PDMS posts ranging 6-20 $\mu \mathrm{m}$ in diameter were developed from replica molding by casting an elastomer over a silicon master and spin coating to a $150 \mu \mathrm{m}$ film. Confocal image of cells being compressed by the cytoindentor. Dark circles represent areas of the cells being compressed by PDMS posts.

micropost arrays with customized geometries and densities can be easily prototyped, making CLiC a versatile cytoindentation tool for manipulating and visualizing cells. Distortions to cell shape in vitro have been linked to activation of apoptosis (14), and it remains unclear whether live cells can withstand sustained local loading. To demonstrate that the CLiC cytoindentor does not cause apoptosis or cytotoxicity, cells were indented for extended periods of time while cell lysis and oxidative stress levels were measured.

\section{Materials and methods}

Cell culture

Experiments were conducted on Chinese hamster ovary ( $\mathrm{CHO}-\mathrm{K} 1)$ cells stably expressing paxillin fused with enhanced green fluorescent protein (EGFP). Cells were cultured in Dulbecco's modified Eagle medium (DMEM) supplemented with non-essential amino acids, penicillinstreptomycin, $10 \%$ fetal bovine serum (FBS), $0.5 \mathrm{mg} / \mathrm{mL}$ geneticin (for maintenance of selection), and buffered with 25 mM HEPES. All cell culture reagents were from Gibco, Paisley, UK.

For cytoindentation, $\mathrm{CHO}-\mathrm{K} 1$ cells were plated on 35-mm coverglass bottom dishes (Cat\#FD-35; World Precision Instruments, Sarasota, FL). Dishes were coated with $2 \mu \mathrm{g} / \mathrm{mL}$ human plasma fibronectin (Millipore, Temecula, CA) for $45 \mathrm{~min}$ at $37^{\circ} \mathrm{C}$. Dishes were then washed three times with PBS to remove excess fibronectin before plating cells. Cells were plated and allowed to adhere for $12 \mathrm{~h}$ at $37^{\circ} \mathrm{C}$ in a $5 \%$ humidified $\mathrm{CO}_{2}$ environment prior to cytoindentation.

\section{Confocal microscopy}

$\mathrm{CHO}-\mathrm{K} 1$ cells were imaged on a Carl Zeiss Axiobserver microscope (Jena, 
Germany) using a plan-Apochromat $20 \times / 0.8$ M27 air objective coupled to a 710 laser scanning confocal microscope head. Two different instrument settings were used for cell tracking and functional imaging experiments.

For cell tracking experiments, the triple color main beam splitter (MBS 488/561/633) was used, and paxillinEGFP fluorescence was excited using $2 \%$ power from the $488 \mathrm{~nm}$ laser line of a 25-mW argon ion laser. The photomultiplier tube (PMT) voltage was maintained at $800 \mathrm{~V}$, while the pinhole size was set to $38 \mu \mathrm{m}$ or 1 Airy Unit. Images were collected using an $850 \times 850 \mu \mathrm{m}$ sub-region at $1024 \times$ 1024-pixel resolution with a $1.27 \mu s$ pixel dwell time and $2 \times$ line averaging. To track cells post-compression, 3 sets of XY-tile scan Z stacks were collected over a range of $\sim 10 \mu \mathrm{m}$ in height, with 13 image planes and 0.837 $\mu \mathrm{m}$ between $Z$ sections. Z stacks were collected every $30 \mathrm{~min}$ for $12 \mathrm{~h}$ at 2 separate locations, and these images in the xy-planes were stitched together by the Zeiss Zen software.

To image mitochondrial membrane potential, a two-color main beam splitter (MBS 488/543) was used, and tetramethyl rhodamine methyl ester (TMRM) (Molecular Probes, Eugene OR) was excited using $2 \%$ power from a $543 \mathrm{~nm}$ HeNe laser line. The PMT voltage was maintained at $800 \mathrm{~V}$, while the pinhole size was set to $116 \mu \mathrm{m}$. Images were collected using a $415 \times$ $415 \mu \mathrm{m}$ sub-region at $1024 \times 1024-$ pixel resolution, $1.27 \mu$ s pixel dwell time, and $16 \times$ line averaging. Three sets of images were collected every $60 \mathrm{~s}$ for 10 iterations.

\section{CLiC cytoindentor}

A customized CLiC system (MadCityLabs Inc., Madison, WI) was designed to hold a coverslip-bottomed 35-mm cell culture dish. The dish was centered with respect to the CLiC lens (ThorLabs, Newton, NJ), and a field-of-view of cells was chosen by fine translation of the $35-\mathrm{mm}$ dish in the $x y$-plane using two micrometers (8 mm range; 5/64" pitch; ThorLabs, Newton, NJ) (Figure 1A). A plexiglass enclosure housed the entire device, and a stage-top incubator system [Live Cell Instruments (LCI), Seoul, Korea]

A

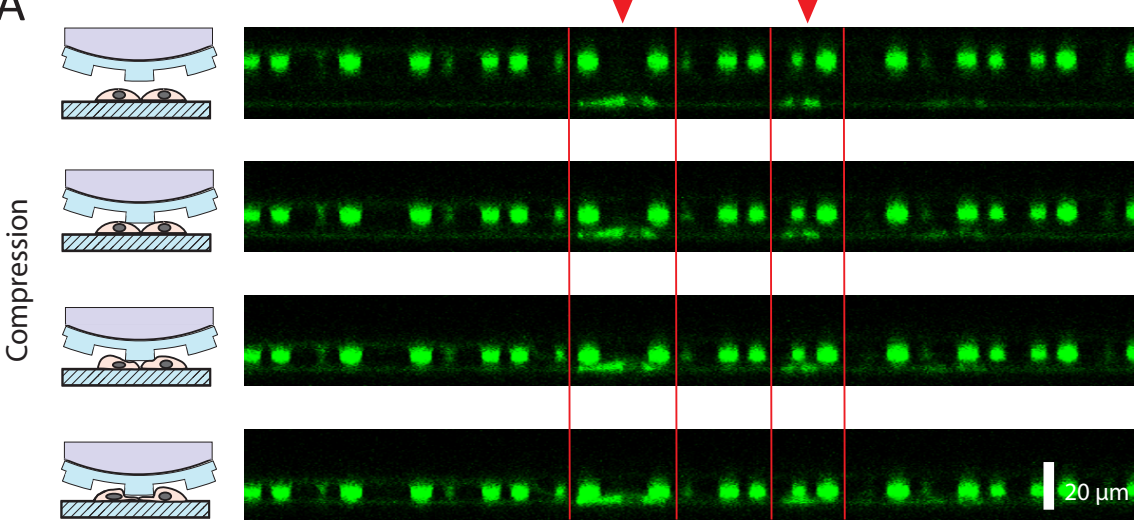

B
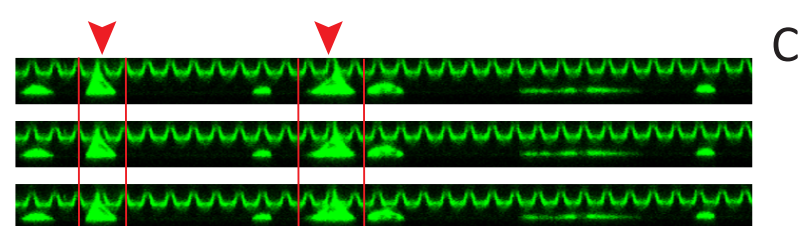

둥

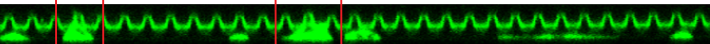

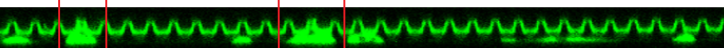

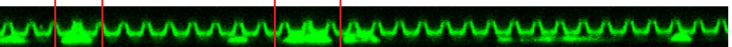

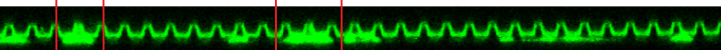

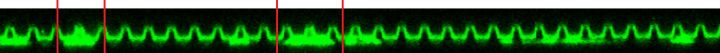

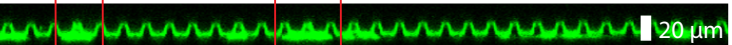

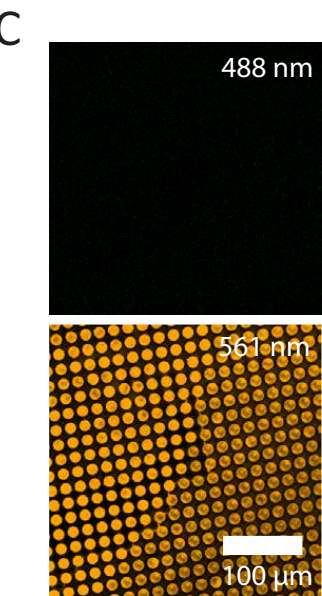

Figure 2. Demonstration of convex lens-induced confinement (CLiC) cytoindentation of cells with polydimethylsiloxane (PDMS) posts. (A) Confocal image sequence projected along the $\mathrm{xz}$-axis of localized compression of Chinese hamster ovary (CHO-K1) cells (red columns) expressing paxillin-EGFP (bottom, green) with PDMS posts (top, green), lowered incrementally at submicron steps until contact was made. (B) Sequence of cell retraction with PDMS posts functionalized with fibronectin, adhering to cells and applying tensile force as the CLiC lens is moved upward. (C) Optically transparent PDMS posts labeled with 1,1'dioleyl-3,3,3',3'tetramethylindocarbocyanine methane sulfonate (Dil). With wide emission filters in place, the Dil is visible in the green channel simultaneously with the EGFP. When imaged with $488 \mathrm{~nm}$ excitation and narrow emission filters, the posts are not visualized in the green channel. With 561 $\mathrm{nm}$ excitation, the Dil-coated PDMS posts are readily visible in the orange emission channel.

was heated to $37^{\circ} \mathrm{C}$ and maintained with humidified $5 \% \mathrm{CO}_{2}$ balanced with air. This instrument design will allow the CLiC cytoindentation setup to be simply modified for compatibility with various stage designs.

An automated MicroStage z-axis stepper motor (MadCityLabs Inc.) provided the driving force of the CLiC-lens, with a step size of 95 $\mathrm{nm}$ and a range of $1.54 \mathrm{~mm}$ (Figure 1A). Sub-micron resolution enabled the precise indentation of living cells with no cell lysis. LabView code was written to computer control the device and enabled precise indentation and retraction, measured using the $x z$-scan feature of the confocal microscope (Figure 2A).

\section{Microfabrication of the} PDMS micropost array

A suite of PDMS micropost arrays were micro-fabricated by replica-molding (15). The replica-molding template was made by first priming a 6 -inch silicon wafer in a vacuum cure oven (Yield Engineering Systems, Livermore, CA) for $30 \mathrm{~min}$. A permanent epoxy negative photoresist (SU-8 2015) was then deposited on the wafer, and the thickness of SU-8 was reduced to 20 $\mu \mathrm{m}$ by spin coating at $2000 \mathrm{rpm}$ for 30 s (McGill Nanotools Microfab, Montreal, 

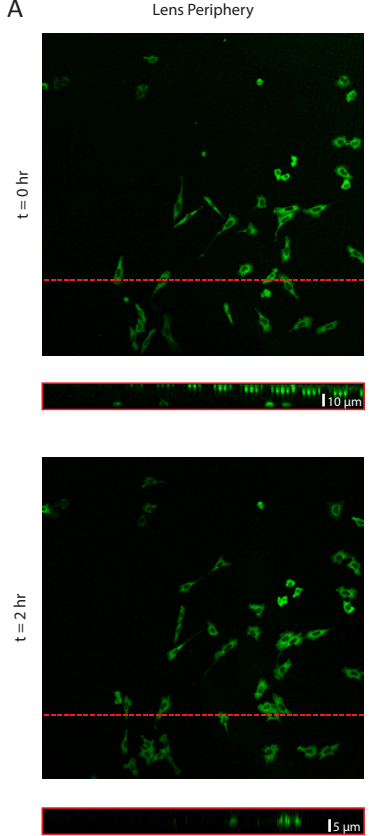

Lens Center
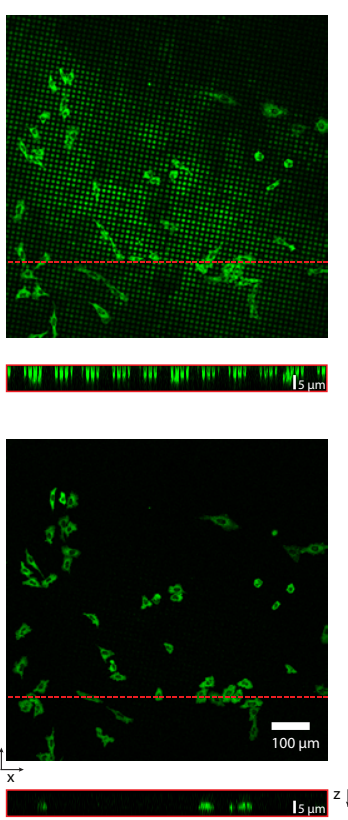

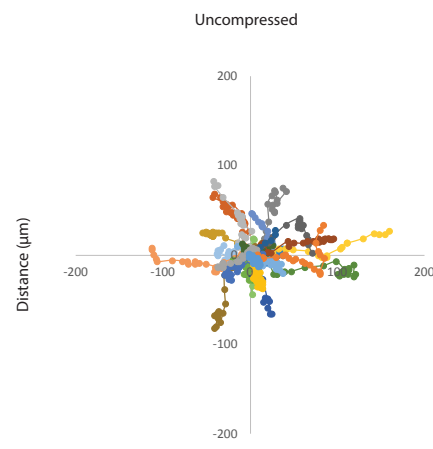

C

Distance ( $\mu \mathrm{m})$

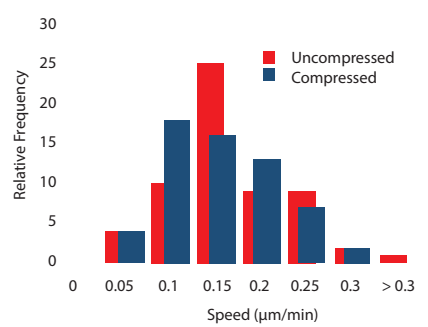

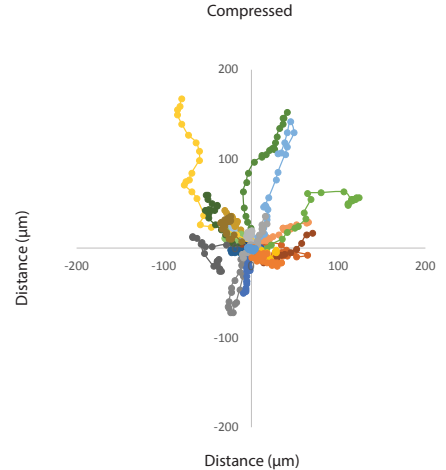

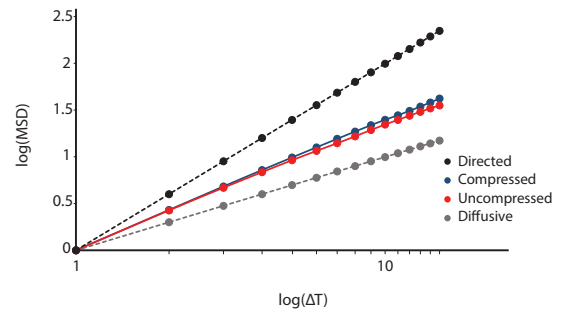

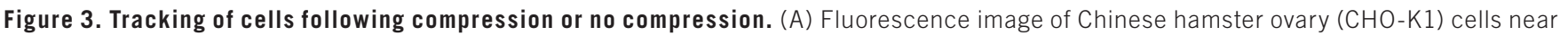

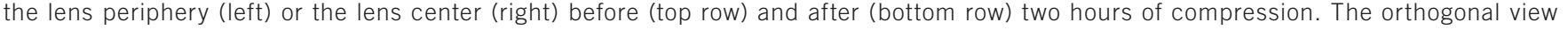

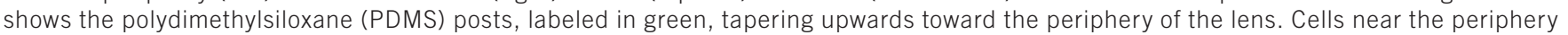

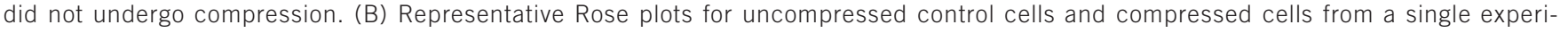

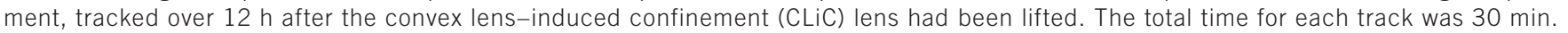

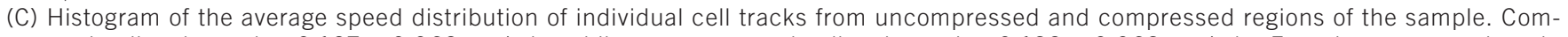

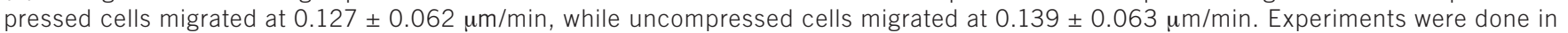

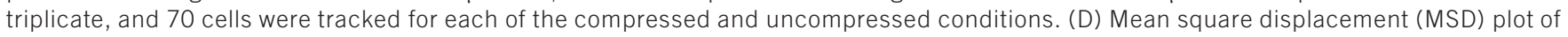

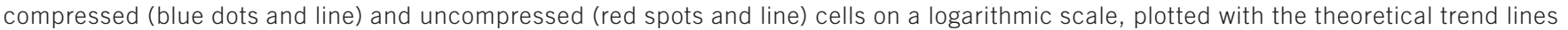

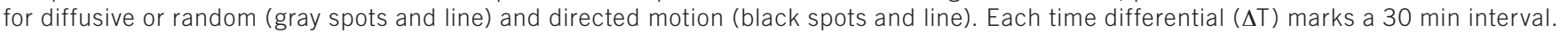

Canada). After soft-baking for 3 min at $95^{\circ} \mathrm{C}$, the wafer was exposed to UV light under a photomask printed with arrays of circles ranging 6-20 $\mu \mathrm{m}$ in diameter, separated by a space of 6 $\mu \mathrm{m}$, and spanning a $10 \mathrm{~mm}$ diameter array. Areas of SU-8 that were not exposed to UV-light were rinsed away by an SU-8 developer for 4 min while the array of cylindrical pits on the silicon wafer remained.

The micropost array template was silanized with trichloro(1,1,2,2,perfluorooctyl)silane (Sigma Aldrich, Milwaukee, WI) prior to casting PDMS films in order to facilitate PDMS release. PDMS micropost arrays were fabricated by pouring a PDMS prepolymer (Sylgard 184) (Dow Corning, Midland, MI) at a 10:1 base to catalyst ratio over the template. The PDMS was degassed under vacuum to remove bubbles, spun at 800 rpm for $20 \mathrm{~s}$ to produce a uniform layer $150 \mu \mathrm{m}$ in thickness (16), and baked at $100^{\circ} \mathrm{C}$ for 30 min to promote crosslinking of the pre-polymer. Using tweezers, the PDMS films with arrays of micropost extrusions were removed from the template, sterilized in 100\% ethanol, and stored in Petri dishes.

\section{Functionalization of PDMS micropost array}

To adhere to cells and enable cytopulling, PDMS micropost arrays were incubated with $50 \mu \mathrm{g} / \mathrm{mL}$ of fibronectin in PBS for $1 \mathrm{~h}$ to coat the posts with fibronectin (17). Cells were cytoindented for 15 min before the stepper motor was used to lift the lens upwards and pull on cells (Figure 2B).

PDMS is an optically transparent elastomer and exhibits little autofluorescence. To visualize the position of microarray posts relative to the cells during confocal microscopy, the micropost arrays were fluorescently labeled with a lipophilic dye, Dil (1,1'dioleyl-3,3,3',3'tetramethylindocarbocyanine methane sulfonate) (Invitrogen, Carlsbad, CA). Dil was then imaged with 561-nm laser excitation (Figure 2C). With standard image acquisition settings, Dil and EGFP can be imaged indepen- dently. However, Dil is weakly excited by the $488 \mathrm{~nm}$ laser. If the emission wavelengths for the green fluorescence are expanded, the Dil fluorescence can be imaged simultaneously with the EGFP (Figures 1B and 2B). However, a narrow set of green emission wavelengths could be used if the signals need to be separated with the PDMS micropost array signal in the orange channel (Figure 2C) and the fluorescence emission from EGFP in the green channel.

\section{Cell tracking post-cytoindentation}

The center of the lens, which is the first point of contact with the cells, was located by first lowering the lens into a $35-\mathrm{mm}$ dish containing a solution of $10^{-7} \mathrm{M}$ rhodamine (Sigma Aldrich). The compression lens confines dye molecules to a thin focal plane, and the gap height varies parabolically with the curvature of the lens (12). Regions of low gap height, namely at the center of the lens, exclude more dye molecules; thus, the point of lowest 
A

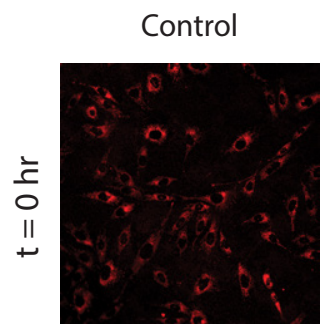

Compressed
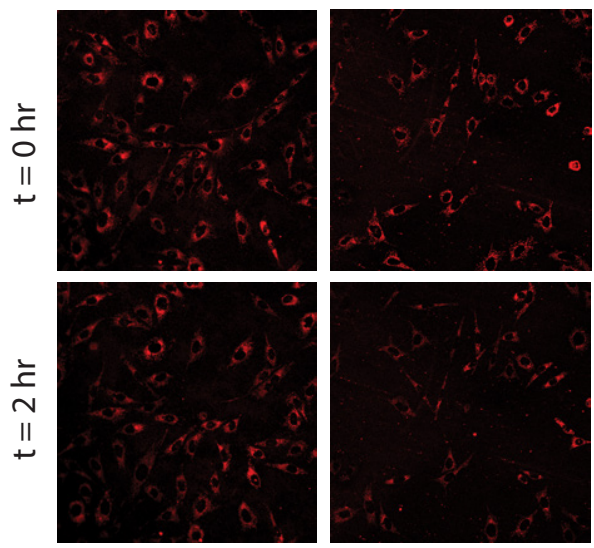

B

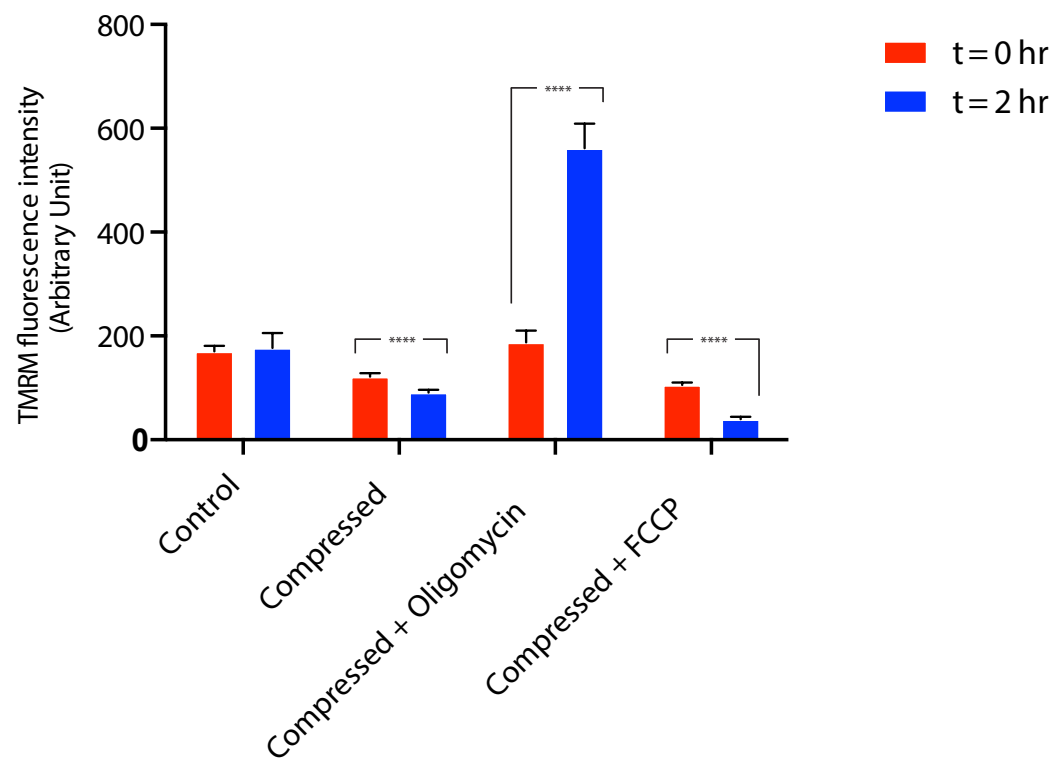

Figure 4. Functional imaging of Chinese hamster ovary (CHO-K1) cells loaded with the potentiometric mitochondrial membrane dye tetramethyl rhodamine methyl ester (TMRM). (A) TMRM intensity in control cells and cells before and after 2 hours of compression. Cells before and after compression were also treated with oligomycin to hyperpolarize the mitochondrial membrane or with carbonyl cyanide-4-(trifluoromethoxy)phenylhydrazone (FCCP) to depolarize the mitochondrial membrane. The oligomycin image after 2 hours of compression appears to be saturated; however, this is just because the intensity was very high, and all of the images in (A) were displayed with the same background, brightness, and contrast settings for an ideal comparison of intensities. (B) Quantification of TMRM intensity data. Experiments were done in triplicate and average intensity measurements were made from 40-45 cells for each condition.

and corrected for the average background intensity. The fluorescence intensity within a $5 \mu \mathrm{m}$ diameter region of interest was then measured for 15 randomly chosen cells and averaged.

\section{Results and discussion}

An automated CLiC device was customized to cytoindent within a live-cell stage-top incubation chamber, designed for a 35-mm cell culture dish. The CLiC compression lens was coated with a custom-designed film covered with an array of $8 \mu \mathrm{m}$ diameter PDMS posts (Figure 1, A and B). The CLiC lens could be centered in the $35 \mathrm{~mm}$ dish and moved along the z-axis with micron precision to locally compress cells with the PDMS posts. Profile $x z$-axis images of cells were collected during incremental indentation and retraction cycles with the PDMS micropost array enhanced CLiC cytoindentation device (Figure 2, A and B). Images of $\mathrm{CHO}-\mathrm{K} 1$ cells expressing paxillinEGFP within adhesions and within the cytosol, before and after indentation, show that cells can undergo localized compression without any evidence of lysis (Figure 2, A and B); however, flattening entire cells with a bare compression lens did cause cell rupture (not shown). Precision in the area of compression in the $x y$-plane is equally important as sensitivity in the $Z$ actuation. By localizing the indentation area to a small subcellular region defined by the size of the PDMS post and its position relative to the cell, the cytosolic volume excluded underneath the PDMS post was small enough to be accommodated by the elasticity of the cell membrane, avoiding lysis. PDMS posts were fluorescently labeled with Dil and could be imaged simultaneously with paxillin-EGFP within the cells (Figure 2, A and B) or exclusively in the orange fluorescence emission channel (Figure 2C).

The plano-convex indentation surface confers several advantages for cell compression. If a flat indentation surface were tilted with respect to the $x y$-plane it would contact the cell culture plate edge-first, while a curved indentation surface would make a uniform contact even if the lens were slightly tilted with respect to the cell culture dish. This makes the curved CLiC cytoindentor platform both robust and practical. Applying CLiC as a cytoindentation technique eliminates the need of a correcting tilting table, which can be complex and expensive (8). Importantly, a convex lens with a large radius of curvature ensures that the surface of the lens center, which is in contact with cells, is locally flat. The height of the ceiling is governed by:

$$
h=\frac{1}{2} \times \frac{r^{2}}{R}
$$

[Eq. 2]

where $r$ is the distance from the point of contact, $R$ is the radius of curvature of the lens, and $h$ is the gap height (12). Here we used a convex lens with 
a focal length of $100 \mathrm{~mm}$, which was essentially flat over a large field-of-view due to the ceiling height being only 25 $\mathrm{nm}$ at $100 \mu \mathrm{m}$ away from the center of lens (Figure 2, A and B). Although the variation in lens height was marginal near the center of the lens, it became markedly larger much further away (>400 $\mu \mathrm{m})$ as $h$ scales exponentially with radius $r$. Thus, the parabolic ceiling of the lens engaged a mild gradient of confinement, which compressed cells near the center of the lens and tapered off at the edge of the lens (Figure 3A, edge of the field-of-view). This heterogeneous cell compression across a large field-of-view allows for control and compression experiments to be conducted as a single experiment in one region of a sample (Figure $3 \mathrm{~A}$ ). Variable compression can be easily measured from the comparison between a 3-D confocal stack of images collected both before and after compression.

The current setup exhibited little backlash and drift. Retracting the lens did not displace any cells in the fieldof-view (Figure 2A), and compressed cells maintained their original position. Thus, cytoindentation with PDMS micropost arrays can be explored as a possible alternative to fixing agents used in super-resolution microscopy techniques (e.g., STORM) to hold cells in place during imaging. The current installment of the cytoindentor relies on visual feedback updated in the $x z$-plane to maneuver the PDMS posts. The axis of cross-section influences the appearance of the PDMS posts. Whereas an $x z$-plane taken near the surface of the posts gives the PDMS posts a solid appearance (Figure 2A), an $x z$-plane taken through the diameter of the posts gives the PDMS posts a hollow appearance (Figure 2B). Because the axial resolution is especially limited on confocal microscopes, precise quantitative measurement of confinement depth per individual cell remains a challenge. It would be interesting to apply the device using structured illumination microscopy to improve z-axis resolution. Additionally, coverage of compression and heterogeneity of compression from cell to cell is complicated by a static distribution of microposts. During cytoindentation, some cells can be indented by more than one micropost, and others can be indented at a location different from other cells. A future cytoindentor design with a closed-loop piezo controller providing positional feedback could report precise indentation depth. Uniform coverage of cells can be addressed by prototyping silicon masters and corresponding PDMS micropost arrays of varying density, and pairing that setup with ideal cell confluency to engineer the number of posts per cell in a precise manner. Patterning of extracellular matrix (ECM) proteins on the coverglass surface could also be paired with patterned micropost arrays. The parallel nature and statistical power of CLiC cytoindentation will allow data to be separated into populations of cells that are perturbed in similar ways. The high-throughput nature of the device will provide tremendous amounts of information that is not readily accessible with existing technologies.

We were also able to demonstrate that the cytoindentor can be used as a cytopuller. PDMS microposts were functionalized with the ECM protein fibronectin and were brought into contact with cells. After 15 min, the cells adhered to the fibronectin-coated posts and could be pulled vertically during lens retraction (Figure $2 \mathrm{~B}$ ). This can be used as a tool to shed new light on how extracellular forces regulate intracellular machinery, such as actin stress fibers.

To test if the cytoindentation had any adverse effects on cell health, cell migration was measured before and after compression. Past work has demonstrated that cell migration speeds slow down when cells are stressed (unpublished data). Our results show that cells continued to migrate even after compression for 2 hours (0.127 $\pm 0.062 \mu \mathrm{m} / \mathrm{min}$ ) (Figure 3, B and C), albeit slightly slower than their uncompressed cohort $(0.139 \pm 0.063 \mu \mathrm{m} / \mathrm{min}$; $P=0.003)$. Visual inspection revealed no obvious morphological changes in the cells, such as rounding up or blebbing. Cell migration appeared to be more directional following compression as seen in the representative Rose plots (Figure 3B). However, an MSD analysis of the data revealed that the type of cell movement was similar before or after compression (Figure 3D). The black curve shows the expected MSD curve for directed cell movement, the gray curve for random movement of the cells, and the blue and red plots for compressed and uncompressed cells, respectively (Figure 3D). The slopes of the 2 plots are nearly identical at 1.31 and 1.37, respectively. Approximately $14 \%$ of compressed cells and uncompressed control cells exhibited directed motion. The fact that indented cells continued to migrate post-compression and behave similar to their uncompressed counterparts suggests that the CLiC cytoindentation does not damage membrane integrity nor does it induce significant cell stress. CLiC cytoindentation thus serves as a viable platform for high-throughput, parallelized local loading on live cells.

We did want to look for more subtle indications of cell stress, so we examined the oxidative stress response using the mitochondrial potential marker TMRM, a potential-driven colorimetric dye. Whereas TMRM accumulates in the inner membrane space of the mitochondria of healthy cells, TMRM is released into the cytosol and does not fluoresce during cell stress or apoptosis when the mitochondrial membrane depolarizes (18). Thus, TMRM is a sensitive indicator of changes in mitochondrial membrane potential resulting from mild cell stress. While TMRM intensity did not change over 2 hours in uncompressed control cells, a small 16\% decrease in TMRM staining intensity was seen following 2 hours of compression. In contrast, depolarization of the mitochondrial membrane with FCCP resulted in a $62 \%$ decrease in fluorescence intensity, and hyperpolarization with oligomycin produced a $225 \%$ increase in fluorescence intensity (Figure 4, A and B). These data suggest compression may induce slight oxidative stress on the cells, but the effect is not drastic.

We have demonstrated that the CLiC cytoindentor does not cause significant changes in cell health. Therefore, it can be used to actuate precise and gentle forces on live cells. This force readout can be inferred by likening the cell to an elastic spring and calculating applied force as a function of displacement. The viscoelastic properties of both $\mathrm{CHO}-\mathrm{K} 1$ cells and PDMS have been well-characterized in the literature. 
AFM measurements of the elastic moduli of living $\mathrm{CHO}-\mathrm{K} 1$ cells report a range of 244-1020 $\mathrm{Pa}$ (19), while the elastic moduli of PDMS composed of 10:1 curing agent to pre-polymer range 1.93-2.27 MPa (20). Both report a Poisson ratio of $\sim 0.5$, which assumes both materials are incompressible. By modeling the cells and the PDMS indenter posts as spherical elastic surfaces (21), the estimated force actuated on the cells by every rod is $\sim 110 \mu \mathrm{N}$ with the CLiC cytoindentor.

The CLiC cytoindentor can serve as a tunable platform for high-throughput, parallelized local loading on live cells. The CLiC lens can be enhanced with micropatterned polydimethylsiloxane (PDMS) films functionalized to either compress or pull on multiple cells and can be customized to confine subcellular compartments under various geometries. We also envision the PDMS micropost array could serve as a barrier around which cells must migrate instead of being pinned down by the microposts. Studies have shown that cells confined to narrow channels and varying densities of ECM migrate more quickly $(22,23)$. PDMS microposts of varying densities and geometries could be rapidly prototyped to conduct similar studies of confined cells. With no apparent signs of apoptosis induction or significant cell stress, the CLiC cytoindentor can be used as a cell perturbation tool to investigate force-dependent cell mechanochemistry, among other phenomena. Subsequent integration of this device with biophysical tools such as fluorescence correlation spectroscopy, which measures diffusing and interacting biomolecules, could enable study of the chemo-mechanical effects of the applied load at the single-adhesion or single-molecule level.

\section{Author contributions}

C.M.B. and S.R.L. designed the research project and supervised the work by B.J.; B.J., D.J.B., and S.R.L. designed and built the prototype tools; B.J., A.M., and D.J. fabricated the micropost arrays; B.J., T.L.W., and C.G.B. performed the cell migration and TMRM live-cell experiments; B.J., C.G.B., C.M.B., and S.R.L. analyzed the data; and B.J., C.M.B., and S.R.L. designed the figures and wrote the paper.

\section{Acknowledgments}

$\mathrm{CHO}-\mathrm{K} 1$ cells expressing paxillin-EGFP were a kind gift from the laboratory of Alan Rick Horwitz at the University of Virginia. We would like to thank Yan-rung Duh and George Tali for their help in live-cell imaging and cell culture. We also thank Gil Henkin for his insight on analyzing cell migration behavior, Christopher McFaul for his assistance in testing the initial CLiC cytoindentation prototypes, and Seddik Benhammadi and Sasa Ristic for their microfabrication support. We would like to thank Alexander Hofkirchner, François Michaud, Shane Scott, Caleb Guthrie, and Jason Leith for their fruitful discussions of this manuscript, and Monserratt Lopez and Peter Grütter for their helpful suggestions on the theory of biomechanical deformations. Financial support was provided through a National Science and Engineering Research Council of Canada (NSERC) Engage Grant and an Industrial Undergraduate Student Research Award (B.J.) funded in partnership with Quorum Technologies Inc. D.B. received an NSERC Graduate Research Fellowship and financial support through the Bionanomachines CREATE program. Microscopy experiments for this project were performed at the McGill Life Sciences Complex Advanced Biolmaging Facility (ABIF).

\section{Competing interests}

The authors declare no competing interests.

\section{References}

1. Leipzig, N.D. and K.A. Athanasiou. 2005. Unconfined Creep Compression Of Chondrocytes. J. Biomech. 38:77-85.

2. Nguyen, T.D., A. Oloyode, and Y. Gu. 2014. Stress Relaxation Analysis Of Single Chondrocytes Using Porohyperelastic Model Based On AFM Experiments. Theor. Appl. Mech. Lett. 4:054001.

3. Shin, D. and K.A. Athanasiou. 1999. Cytoindentation for Obtaining Cell Biomechanical Properties. J. Orthop. Res. 17:880-890.

4. Lauffenburger, D.A. and A.F. Horwitz. 1996. Cell Migration: a Physically Integrated Molecular Process. Cell 84:359-369.

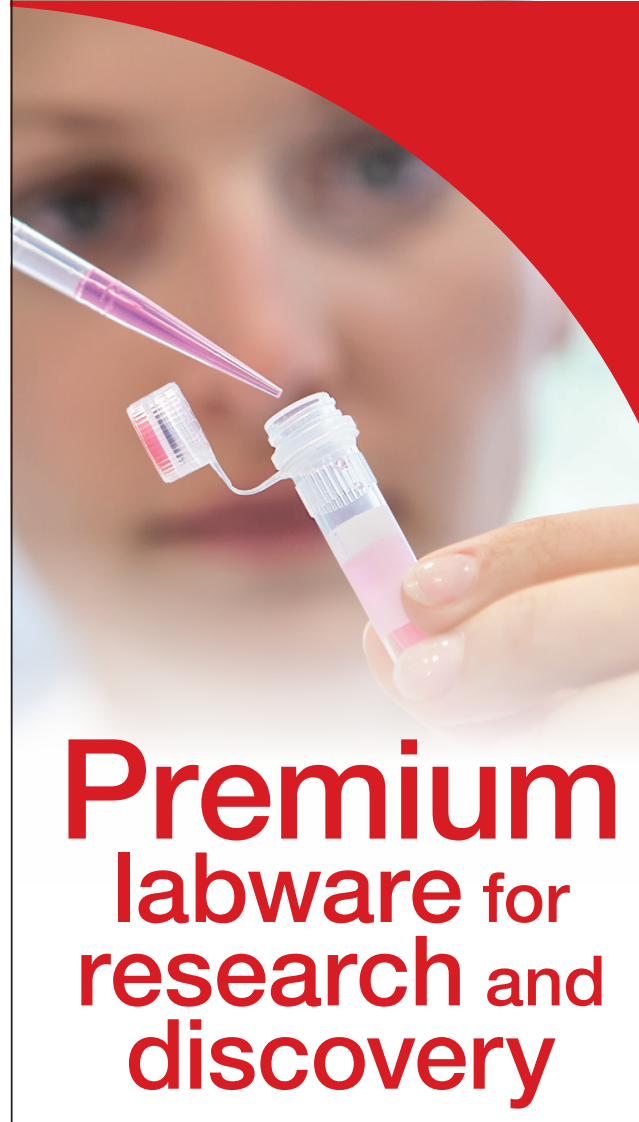

Screw Cap Micro Tubes

- Heat, freeze, store and more

- High centrifugation limits

- 3 purity standards for sensitive applications

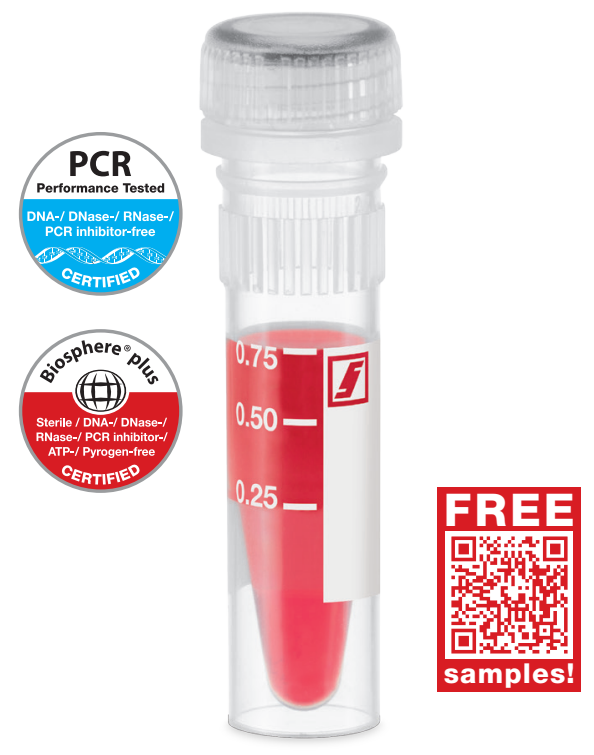

www.sarstedt.com 
5. Geiger, B., J.P. Spatz, and A.D. Bershadsky. 2009. Environmental Sensing Through Focal Adhesions. Nat. Rev. Mol. Cell Biol. 10:21-33.

6. Buxboim, A., I.L. Ivanovska, and D.E. Discher. 2010. Matrix Elasticity, Cytoskeletal Forces And Physics Of The Nucleus: How Deeply Do Cells 'Feel' Outside And In? J. Cell Sci. 123:297-308

7. Franz, C.M. and D.J. Muller. 2005. Analyzing Focal Adhesion Structure by Atomic Force Microscopy. J. Cell Sci. 118:5315-5323.

8. Peeters, E A., C.V. Bouten, C.W. Oomens, and F.P. Baaijens. 2003. Monitoring the Biomechanical Response of Individual Cells Under Compression: a New Compression Device. Med. Biol. Eng. Comput. 41:498-503.

9. Danilowicz, C., D. Greenfield, and M. Prentiss. 2005. Dissociation of LigandReceptor Complexes Using Magnetic Tweezers. Anal. Chem. 77:3023-3028.

10. Lee, L.M. and A.P. Liu. 2015. A microfluidic pipette array for mechanophenotyping of cancer cells and mechanical gating of mechanosensitive channels. Lab Chip 15:264-273.

11. Sasoglu, F.M., A.J. Bohl, K.B. Allen, and B.E. Layton. 2009. Parallel Force Measurement with a Polymeric Microbeam Array Using an Optical Microscope and Micromanipulator. Comput. Methods Programs Biomed. 93:1-8.

12. Leslie, S.R., A.P. Fields, and A.E. Cohen. 2010. Convex Lens-Induced Confinement for Imaging Single Molecules. Anal. Chem. 82:6224-6229.

13. Berard, D., C.M.J. McFaul, J.S. Leith, A. Arsenault, F. Michaud, and S.R. Leslie. 2013. Precision Platform for Convex LensInduced Confinement Microscopy. Review of Scientific Instruments. 84:103704.

14. Chen, C.S., M. Mrksich, S. Huang, G.M. Whitesides, and D.E. Ingber. 1997 Geometric Control of Cell Life and Death. Science 276:1425-1428.

15. Xia, Y. and G.M. Whitesides. 1998. Soft Lithography. Angew. Chem. Int. Ed. 37:550575 .

16. DiLuzio, W.R., L. Turner, M. Mayer, P. Garstecki, D.B. Weibel, H.C. Berg, and G.M Whitesides. 2005. Escheria coli swim on the right-hand side. Nature 435:1271-1274.

17. Yang, M.T., J. Fu, Y.K. Wang, R.A. Desai, and C.S. Chen. 2011. Assaying Stem Cell Mechanobiology on Microfabricated Elastomeric Substrates with Geometrically Modulated Rigidity. Nat. Protoc. 6:187-213.

18. Scaduto, R.C. and L.W. Grotyohann. 1999. Measurement Of Mitochondrial Membrane Potential Using Fluorescent Rhodamine Derivatives. Biophys. J. 76:469-477.

19. Zhao, M., C. Srinivasan, D.J. Burgess, and B.D. Huey. 2006. Rate- and Depth-Dependent Nanomechanical Behavior of Individual Living Chinese Hamster Ovary Cells Probed by Atomic Force Microscopy. J. Mater. Res. 21:1906-1912.

20. Johnston, I.D, D.K McCluskey, C.K.L. Tan, and M.C. Tracey. 2014. Mechanical Characterization of Bulk Sylgard 184 for Microfluidics and Microengineering. J. Micromech. Microeng. 24:035017.

21. Puttock, M.J. and E.G. Thwaite. 1969. Elastic Compression of Spheres and Cylinders at Point and Line Contact, National Standards Laboratory Technical Paper No. 25. Commonwealth Scientific and Industrial Research Organization, Melborne, Australia.

22. Balzer, E.M., Z. Tong, C.D. Paul, W.C. Hung, K.M. Stroka, A.E. Boggs, S.S. Martin, and K. Konstatopoulos. 2012. Physical confinement alters cell adhesion and migration phenotypes. FASEB J. 26:4045-4056.

23. Pathak, A. and S. Kumar. 2012. Independent regulation of tumor cell migration by matrix stiffness and confinement. Proc. Natl. Acad. Sci. USA 109:10334-10339.

Received 04 September 2015; accepted 18 March 2016

Address correspondence to Sabrina R. Leslie, Department of Physics, McGill University, Montreal, Quebec Canada. E-mail: sabrina.leslie@mcgill.ca

To purchase reprints of this article, contact: biotechniques@fosterprinting.com

\section{mong Improve Your PCR Workflow}

\section{DNA Rapid Extract" Kit}

\author{
FAST • SIMPLE • EFFICIENT
}

\section{Diffinity DNA RapidExtract ${ }^{\mathrm{TM}}$ Kit}

Our DNA RapidExtract ${ }^{\mathrm{TM}}$ Kit lyses DNA in a single incubation step and yields PCR-ready DNA in 10 minutes.

\section{ONE KIT for multiple sample types}

$\begin{array}{lll}\text { - Plant (leaf, seeds, pulp) } & \text { - Hair Follicle } & \text { - Bird Feather } \\ \text { - Blood (EDTA or heparin) } & \text { - Animal Tissue } & \bullet \text { Feces }\end{array}$

- Buccal Swab

Easy-to-use protocol for multiple sample types

No special storage is required

KaneKa

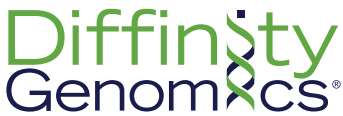

\section{Purify}

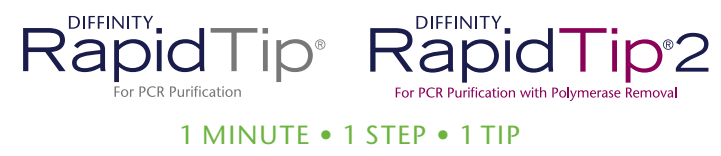

Diffinity RapidTip ${ }^{\circledR}$ and RapidTip ${ }^{\circledR}$

Diffinity Genomics ${ }^{\circledR}$ RapidTips remove dNTPs, primers, primer-dimers and DNA polymerase from your PCR sample while providing highrecovery of desired DNA fragments for downstream applications (genotyping, T-A cloning, and Sanger cycle sequencing reactions).

No bind-wash-elute, enzymes, or magnetic beads

Each tip is pre-packed with Differentially Functionalized Nano-Porous Particles for use right out of the box

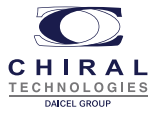

